Pacific

Journal of

Mathematics

REMOVABLE SINGULARITIES FOR YANG-MILLS CONNECTIONS IN HIGHER DIMENSIONS

BAOZHONG YANG 


\title{
REMOVABLE SINGULARITIES FOR YANG-MILLS CONNECTIONS IN HIGHER DIMENSIONS
}

\author{
BAOZHONG YANG
}

\begin{abstract}
We prove several removable singularity theorems for singular Yang-Mills connections on bundles over Riemannian manifolds of dimensions greater than four. We obtain the local and global removability of singularities for Yang-Mills connections with $L^{\infty}$ or $L^{\frac{n}{2}}$ bounds on their curvature tensors, with weaker assumptions in the $L^{\infty}$ case and stronger assumptions in the $L^{\frac{n}{2}}$ case. With the global gauge construction methods we developed, we also obtain a 'stability' result which asserts that the existence of a connection with uniformly small curvature tensor implies that the underlying bundle must be isomorphic to a flat bundle.
\end{abstract}

\section{Introduction.}

Uhlenbeck's original paper [11] on removing isolated singularities of YangMills connections on four manifolds is important not only in its applications in the compactification of the moduli space of self-dual connections on Riemannian four manifolds, but also in the analytic techniques introduced in it. Later on, there has been much work on the removable singularities for Yang-Mills connections. Some of these work focused on the case of isolated singularities for connections in different dimensions and possibly coupled with a section (a Higgs field), such as $[\mathbf{1}],[4],[6]$ and [7]. Some other works treated more general singularities, such as [5], [3], [8] and [10]. The fundamental work of Tian in [10] on the analysis of Yang-Mills connections in higher dimensions gave some guidance on what we should expect about the singularities of Yang-Mills connections on manifolds of dimensions greater than 4. It turns out that if we are considering connections within the compactification of smooth Yang-Mills connections on an $n$-dimensional manifold, then the most general type of singularities to start with is probably an $H^{n-4}$-rectifiable closed set. While the most general removable singularity theorem in higher dimensions has not been proved yet, this paper is an effort to understand the removable singularities and the related gauge problems in higher dimensions.

We shall assume that all the vector bundles in this paper have a compact structure group $G$ and all connections and gauge transformations refer to 
$G$-objects. We fix a metric on $G$ by embedding $G$ into some orthogonal group and denote by Id the unit element of $G$.

Before stating our main results in this paper, we shall first clarify the meaning of removable singularities we are going to use here. Because of the technical difficulty of defining suitable concepts of weak solutions to the Yang-Mills equations, instead of considering regularity theories for weak solutions (as in the case of harmonic maps and some other nonlinear PDE problems), people usually consider removable singularity theorems in the following (or similar and slightly different) context: We shall usually consider a Yang-Mills connection $A$ on a vector bundle $E$ on a manifold $M$ with a closed singular set $S$, that is, $A$ is defined and smooth on $M \backslash S$ and $A$ satisfies the Yang-Mills equation on $M \backslash S$. We say that the singularity of $A$ is removable if there exists a vector bundle $E^{\prime}$ on $M$ such that $\phi:\left.E\right|_{M \backslash S} \rightarrow$ $E^{\prime}$ gives an embedding of vector bundles preserving the $G$-structures, and there exists a gauge transformation $g$ of $E$, defined and smooth on $M \backslash S$ such that the connection $g(A)$ is identified under $\phi$ with a connection on $\left.E^{\prime}\right|_{M \backslash S}$, which is the restriction of a smooth connection on $E^{\prime}$ over $M$. We note that if the original connection $A$ is singular on $S$, then the smoothing gauge transformation $g$ must be discontinuous on $S$, hence in general $E^{\prime}$ and $E$ may not be topologically isomorphic as vector bundles. Under a local trivialization of $E$, the connection $A$ may be identified with a $\mathcal{G}$-valued 1 -form, where $\mathcal{G}$ is the Lie algebra of $G$. Then locally the removability of the singularity of $A$ is equivalent to the existence of a $G$-valued function $g$ (which is smooth away from $S$ ) such that $g(A)=g A g^{-1}-d g \cdot g^{-1}$ can be extended to a smooth $\mathcal{G}$-valued 1-form.

In [10, 2.3], an admissible Yang-Mills connection is defined as a Yang-Mills connection $A$ with a closed singular set $S$ such that the $(n-4)$-dimensional Hausdorff measure of $S$ is locally finite and $\mathrm{YM}(A)=\int_{M}\left|F_{A}\right|^{2}<\infty$. The connections we considered in this work are within the class of admissible Yang-Mills connections. Another important notion is the stationarity of a connection, which in particular implies the monotonicity formula for the scaling-invariant Yang-Mills functional, see [10,2.1]. Since we assume $L^{\infty}$ or $L^{\frac{n}{2}}$ boundedness of the curvature in this paper, the connections we consider satisfy the monotonicity for $L^{\frac{n}{2}}$ norm of the curvature trivially, hence we don't need to assume stationarity here.

Our first result is the following local removable singularity theorem for singular connections with $L^{\infty}$ bounds on their curvatures.

Theorem 1. Let $E$ be the trivial bundle over the Euclidean unit cube $U=$ $(0,1)^{n} \subset \mathbb{R}^{n}$ with the standard product metric. Assume that $A$ is an admissible Yang-Mills connection on $E$ with singular set $S$. Then there exists $\varepsilon_{1}=\varepsilon_{1}(n, G)>0$, such that if

$$
\left\|F_{A}\right\|_{L^{\infty}(U)} \leq \varepsilon_{1}
$$

then the singularity of $A$ is removable over $U$. 
We note that there is no extra assumption about the singularity set $S$ except closedness and the dimensional requirement. With some global gauge patching arguments, we have the following global version of the above theorem:

Theorem 2. We assume that $M$ is a compact Riemannian manifold such that all representations $\pi_{1}(M) \rightarrow G$ are the trivial one and $E$ is the trivial smooth bundle over $M$ with a smooth $G$-structure. Assume that $A$ is a Yang-Mills connection on $E$ with singularity set $S$. Then there exists $\varepsilon_{2}=$ $\varepsilon_{2}(M, G)>0$, such that if

$$
\left\|F_{A}\right\|_{L^{\infty}(M)} \leq \varepsilon_{2},
$$

then the singularity of $A$ is globally removable.

We should mention that without the triviality of the bundle $E$, there might not be global smoothing gauges for $A$ even if the singularity of $A$ is locally removable (see the remark at the end of Section 4). Our global patching arguments also yield some 'stability' results which roughly mean that a bundle close to a flat bundle (in some sense) must be flat. In particular, we have:

Theorem 3 (Corollary 2). Assume that $M$ is a compact n-dimensional Riemannian manifold and $E$ is a smooth vector bundle over $M$ with a smooth connection $A$ on it. Then there exists a constant $\varepsilon_{9}=\varepsilon_{9}(M)>0$, such that if

$$
\left\|F_{A}\right\|_{L^{\infty}(M)} \leq \varepsilon_{9}
$$

then $E$ is smoothly isomorphic to a flat bundle.

It might be possible to improve the $L^{\infty}$ norm bounds in the above theorem to some $L^{p}(p<\infty)$ bounds. In the next two theorems, we use the $L^{\frac{n}{2}}$ norm instead of the $L^{\infty}$ norm of the curvature of the connection. We also assume the singularity set to be a manifold.

Assume that $4 \leq k \leq n$ is an integer. Let $B_{1}^{k}$ be the open unit $k$-ball in $\mathbb{R}^{k}$, and $D_{1}=B_{1}^{n-k} \times B_{1}^{k} \subset \mathbb{R}^{n}$ be the Cartesian product of two balls. Assume that $E$ is a trivial vector bundle on $D_{1} \backslash\left(B_{1}^{n-k} \times\{0\}\right)$. We shall consider connections with singularity $B_{1}^{n-k} \times\{0\} \subset D_{1}$. This is the standard local model for connections with singularities being manifolds of codimension at least 4 .

Theorem 4. Assume that $\widetilde{A}$ is a Yang-Mills connection on $E$ with the singularity $B_{1}^{n-k} \times\{0\} \subset D_{1}$. Then there exists a constant $\varepsilon_{3}=\varepsilon_{3}(n, k, G)>0$, such that if

$$
\left\|F_{\widetilde{A}}\right\|_{L^{\frac{n}{2}}\left(D_{1} \backslash\left(B_{1}^{n-k} \times\{0\}\right)\right.} \leq \varepsilon_{3},
$$

then the singularity of $A$ is removable over $D_{\frac{1}{2}}=B_{\frac{1}{2}}^{n-k} \times B_{\frac{1}{2}}^{n-k}$. 
The following global version is a corollary of Theorem 2 and Theorem 4:

Theorem 5. We assume that $M$ is a compact Riemannian manifold such that all representations $\pi_{1}(M) \rightarrow G$ are the trivial one and $E$ is the trivial smooth bundle over $M$ with a smooth $G$-structure. Assume that $A$ is a YangMills connection on $E$ such that the singularity set $S$ of $A$ is a closed smooth submanifold of codimension $\geq 4$. Then there exists $\varepsilon_{4}=\varepsilon_{4}(M, G)>0$, such that if

$$
\left\|F_{A}\right\|_{L^{\frac{n}{2}(M)}} \leq \varepsilon_{4}, \quad \forall x \in M,
$$

then the singularity of $A$ is removable.

We make the assumption that $S$ is a submanifold because we need the good product local model to prove Theorem 4. It is conceivable that this assumption may be relaxed. A conjecture is that we don't need any additional assumption on $S$.

We remark here also that if we allow the constant $\varepsilon_{4}$ in Theorem 5 to depend also on the singularity set $S$, then the conclusion of Theorem 5 follows from the local theorem, Theorem 4 directly without the need of Theorem 2. We also would like to point out that a similar result to Theorem 4 for coupled Yang-Mills-Higgs fields has been proved in Thomas Otway's paper [3]. Our proof of Theorem 4 here is based on the work of Rådo [5] on singular connections on four manifolds with codimension two singularities. After we finished this work, we learned that in a recent work [9], Tao and Tian proved the local removability of singularities for stationary admissible YangMills connections with singularities being manifolds. That will be a stronger result than Theorem 4 here.

In Section 2 we prove Theorem 4 and Theorem 5. In Section 3 we use some local gauge patching techniques to prove Theorem 1. In Section 4, we develop some global gauge patching results, including Theorem 3 and finally prove Theorem 2.

\section{Removable singularities with $L^{\frac{n}{2}}$ norm bounds of curvatures.}

Assume that $3 \leq k \leq n$ is an integer. Let $B_{1}^{k}$ be the open unit $k$-disk in $\mathbb{R}^{k}$, and $D_{1}=B_{1}^{n-k} \times B_{1}^{k} \subset \mathbb{R}^{n}$. Assume that $E \rightarrow D_{1} \backslash\left(B_{1}^{n-k} \times\{0\}\right)$ is a trivial vector bundle.

Theorem 6. There exist constants $\varepsilon_{5}=\varepsilon_{5}(n, k, G)>0$ and $C=C(n, k, G)$ $>0$, such that if $\nabla+A$ is a connection on $E$ with the singularity $B_{1}^{n-k} \times\{0\}$, $A \in L_{1, \mathrm{loc}}^{\frac{n}{2}}\left(D_{1} \backslash\left(B_{1}^{n-k} \times\{0\}\right)\right)$, and $F_{A} \in L^{\frac{n}{2}}\left(D_{1}\right)$, and

$$
\left\|F_{A}\right\|_{L^{\frac{n}{2}}\left(D_{1}\right)} \leq \varepsilon_{5},
$$


then $\nabla+A$ is gauge equivalent, by a gauge transformation in $L_{2, \text { loc }}^{\frac{n}{2}}\left(D_{1} \backslash\right.$ $\left.\left(B_{1}^{n-k} \times\{0\}\right)\right)$ to a connection of the form $\nabla+\widetilde{A}, \widetilde{A} \in L_{1}^{\frac{n}{2}}\left(D_{1}\right)$, and

$$
\|\widetilde{A}\|_{L_{1}^{\frac{n}{2}\left(D_{1}\right)}} \leq C\left\|F_{A}\right\|_{L^{\frac{n}{2}\left(D_{1}\right)}} .
$$

Proof. This theorem is a higher dimensional generalization of Theorem 2.1 in Johan Råde [5]. Actually the setting of the theorem in [5] is even more complicated because Råde considered the possibility of nontrivial holonomy around a codimension 2 singularity. Since the codimension of the singularity here is at least 3 , the complement of the singular set, $D_{1} \backslash\left(B_{1}^{n-k} \times\{0\}\right)$, is simply connected and we can follow the proof in [5] omitting the part involving holonomy to prove the theorem. Of course, since we are in the $n$-dimensional setting, we need to modify the statements of the lemmas and results in [5] (stated in 4-dimensional setting there) accordingly. Mainly we just need to adjust the indices of the various objects and norms. It is not hard to check that the proofs there still work out with these changes and we shall not reproduce the details here.

Proposition 1. An admissible Yang-Mills connection A on a vector bundle $E$ over $M$ is a weak solution of the Yang-Mills equation, i.e.,

$$
\int_{M}\left\langle d_{A} \omega, F_{A}\right\rangle=0
$$

for any $\omega \in C_{c}^{\infty}\left(M, T^{*} M \otimes \operatorname{Ad} E\right)$.

Proof. This type of results are well-known to analysts. For completeness, we give a proof here. Since the question is local, we may assume that $M$ and the singular set $S$ of $A$ are compact. Assume that $H^{n-4}(M)=m<\infty$. For any $\delta>0$, we may find finitely many (geodesic) open balls $B_{r_{i}}\left(x_{i}\right)$ of radii $r_{i}<\delta$, such that $x_{i} \in S, S \subset \cup B_{i}$ and $\sum r_{i}^{n-4} \leq C m$. Choose cutoff functions $\phi_{i}$ such that $\phi_{i}=0$ on $B_{r_{i}}\left(x_{i}\right), \phi_{i}=1$ on $M \backslash B_{2 r_{i}}\left(x_{i}\right), 0 \leq \phi_{i} \leq 1$ on $M$ and $\left|\nabla \phi_{i}\right| \leq C r_{i}^{-1}$ on $M$. Let $\phi=\phi_{\delta}=\inf _{i} \phi_{i}$ on $M$. Then $\phi(x)$ is supported away from $S$ and if we let $N_{2 \delta}(S)=\{y \in M: \operatorname{dist}(y, S) \leq 2 \delta\}$, then $\phi(x)=1$ if $x \in M \backslash N_{2 \delta} S$. We have

$$
|\nabla \phi(x)| \leq \sup _{i}\left|\nabla \phi_{i}(x)\right|, \quad \forall x \in M
$$

Now we have, for any $\omega \in \Omega(\operatorname{Ad} E)$, 


$$
\begin{aligned}
& \int_{M} \phi\left\langle d_{A} \omega, F_{A}\right\rangle \\
& =-\int_{M} \phi \operatorname{tr}\left(d_{A} \omega \wedge * F_{A}\right) \\
& =-\int_{M} d\left(\phi \operatorname{tr}\left(\omega \wedge * F_{A}\right)\right)+\int_{M} d \phi \wedge \operatorname{tr}\left(\omega \wedge * F_{A}\right)+(-1) \int_{M} \phi \operatorname{tr}\left(\omega \wedge d_{A} * F_{A}\right) \\
& =\int_{M} d \phi \wedge \operatorname{tr}\left(\omega \wedge * F_{A}\right), \text { because } A \text { is smooth Yang-Mills away from } S .
\end{aligned}
$$

Now

$$
\begin{aligned}
\left|\int_{M} d \phi \wedge \operatorname{tr}\left(\omega \wedge * F_{A}\right)\right| & \leq C \int_{M}|\nabla \phi|\left|F_{A}\right| \\
& \leq C\left(\int_{N_{2 \delta}(S)}|\nabla \phi|^{2}\right)^{\frac{1}{2}}\left(\int_{N_{2 \delta}(S)}\left|F_{A}\right|^{2}\right)^{\frac{1}{2}} \\
& \leq C\left(\sum_{i} \int_{N_{2 \delta}(S)}\left|\nabla \phi_{i}\right|^{2}\right)^{\frac{1}{2}}\left(\int_{N_{2 \delta}(S)}\left|F_{A}\right|^{2}\right)^{\frac{1}{2}} \\
& =C\left(\sum_{i} \int_{B_{2 r_{i}}\left(x_{i}\right)}\left|\nabla \phi_{i}\right|^{2}\right)^{\frac{1}{2}}\left(\int_{N_{2 \delta}(S)}\left|F_{A}\right|^{2}\right)^{\frac{1}{2}} \\
& \leq C\left(\sum_{i} r_{i}^{n-2}\right)\left(\int_{N_{2 \delta}(S)}^{\left.\left|F_{A}\right|^{2}\right)^{\frac{1}{2}}}\right. \\
& \leq C\left(\int_{N_{2 \delta}(S)}\left|F_{A}\right|^{2}\right)^{\frac{1}{2}} .
\end{aligned}
$$

If we let $\delta \rightarrow 0$, then the last right-hand side goes to 0 by the $L^{2}$ integrability of $F_{A}$. On the other hand

$$
\int_{M} \phi_{\delta}\left\langle d_{A} \omega, F_{A}\right\rangle \rightarrow \int_{M}\left\langle d_{A} \omega, F_{A}\right\rangle
$$

This gives the weak Equation (6).

Remark. We note here that if $A \in L_{1}^{\frac{n}{2}}$ and $A$ satisfies the Yang-Mills equation weakly, then for any gauge transformation $g \in L_{2}^{\frac{n}{2}}, g(A)=g A g^{-1}-$ $d g g^{-1}$ is still a weak solution of the Yang-Mills equation. The reason is as follows: We have $d_{g(A)}^{*} F_{g(A)}=g\left(d_{A}^{*} F_{A}\right) g^{-1}$. By Sobolev embedding theorems, $g, g^{-1} \in L_{2}^{\frac{n}{2}}$ and $d_{A}^{*} F_{A}=0$ in $L_{-1}^{\frac{n}{2}}$ imply that $g\left(d_{A}^{*} F_{A}\right) g^{-1}$ is 
well-defined in $L_{-1}^{p}$, for any $p<\frac{n}{2}$. Hence $d_{g(A)}^{*} F_{g(A)}=0$ in $L_{-1}^{p}$. That implies $g(A)$ satisfies the Yang-Mills equation weakly.

Now we are ready to prove Theorem 4 - the $\varepsilon$-regularity theorem for admissible Yang-Mills connections with $L^{\frac{n}{2}}$ bounds on the curvature.

Proof of Theorem 4. Assume that $\varepsilon_{3}<\varepsilon_{5}$. Then the connection satisfies the assumption of Theorem 6 . We first apply Theorem 6 to the given connection to obtain a $L_{2}^{\frac{n}{2}}$ gauge in which the connection, still denoted $A$, is in $L_{1}^{\frac{n}{2}}$. Now we may apply the existence theorem of Hodge gauges, Theorem 1.3 in Uhlenleck [12] to see that if $\varepsilon_{3}$ is sufficiently small, then after a further $L_{2}^{\frac{n}{2}}$ gauge transformation, we can make the resulting connection, again denoted $A$, to be in the Hodge gauge, i.e.,

$$
d^{*} A=0,
$$

and with the following elliptic boundary condition:

$$
* A=0, \quad \text { on } \partial D_{1} \text {. }
$$

Since the original admissible Yang-Mills connection is a weak solution of the Yang-Mills equation by Prop. 1, and the gauges we have used are all in $L_{2}^{\frac{n}{2}}$, it follows from the previous remark that we have the weak equation,

$$
d_{A}^{*} F_{A}=0 \text {. }
$$

Now (7), (8) and (9) form a uniform elliptic system with $A \in L_{1}^{\frac{n}{2}}\left(D_{1}\right)$. Therefore, by standard elliptic theory, we may obtain higher regularities and the smoothness of $A$ in $D_{1}$. This removes the singularity.

Proof of Theorem 5. If $\varepsilon_{4}$ is sufficiently small, then the assumptions of Theorem 4 are satisfied locally and we have that locally the singularity of $A$ is removable. Since $\left|F_{A}\right|$ is gauge-invariant, we have in particular that $\left|F_{A}\right|^{2}$ is a smooth function on $M$. However, $\left|F_{A}\right|^{2}$ satisfies a Bochner-Weitzenböck formula and hence the following a priori estimates (by Uhlenbeck, see also $[2]):$

$$
\left|F_{A}\right|^{2}(x) \leq C\left(\rho^{-n} \int_{B_{\rho}(x)}\left|F_{A}\right|^{\frac{n}{2}} d v\right)^{\frac{4}{n}}, \quad \forall x \in M .
$$

We remark here that although the a priori estimates are usually stated for smooth (or stationary) Yang-Mills connections and for scaling invariant $L^{2}$ energies $\rho^{4-n} \int_{B_{\rho}(x)}\left|F_{A}\right|^{2} d v$, we have here the bounds on the $L^{\frac{n}{2}}$ energy which is itself scaling invariant. Corresponding to the monotonicity formula used in the proof of the usual a priori estimates, we have trivially that

$$
\int_{B_{\rho}(x)}\left|F_{A}\right|^{\frac{n}{2}} d v \leq \int_{B_{\sigma}(x)}\left|F_{A}\right|^{\frac{n}{2}} d v, \quad \text { if } \rho \leq \sigma .
$$


Hence the proof of the usual a priori estimates (see [2] or [10, 2.2.1]) can be adapted (almost word by word) to give our version (10). We shall not give the details and refer the reader to the references.

By (10), if $\varepsilon_{4}$ is small enough, we have

$$
\left\|F_{A}\right\|_{L^{\infty}(M)} \leq \varepsilon_{2},
$$

and hence Theorem 2 applies to give the global removability of the singularity of $A$.

\section{Removable singularities with $L^{\infty}$ norm bounds of curvatures.}

The essence of the Proof of Theorem 1 is a construction of a smoothing gauge transformation. The method we shall use here is a refinement of Uhlenbeck's method to construct global gauges on compact manifolds used in $\S 3$ of [12]. The idea of this argument is to modify and glue suitable gauges on different patches inductively to obtain a global gauge. Here because we have infinitely many patches, we have to keep a careful track of the gluing procedure to make sure the gauges we obtained are always suitably bounded, thus amenable for further gluing in the induction.

Before giving the proofs, we introduce the following definitions to make the statements simpler. Let $M$ be a Riemannian manifold and let the index set $I$ be either the set of natural numbers or the set $\{1,2, \ldots, n\}$ for some integer $n$.

Definition. Let $c>0$ be a constant and $K>0$ be an integer. We call a countable collection of open subsets of $M,\left\{U_{\alpha}^{i}\right\}_{\alpha \in I, 1 \leq i \leq K}$, a $(c, K)$-uniform nested covering of $M$ if the following conditions are satisfied:

1) $\bar{U}_{\alpha}^{i+1} \subset U_{\alpha}^{i}$, for $1 \leq i \leq K-1$.

2) $M \subset \bigcup_{\alpha \in I} U_{\alpha}^{K}$.

3) $\#\left\{\alpha: x \in U_{\alpha}^{1}\right\} \leq K, \forall x \in M$.

4) The diameters $r_{\alpha}=\operatorname{diam}\left(U_{\alpha}^{1}\right)$ satisfy,

$$
r_{\alpha} \leq c r_{\beta}, \quad \text { if } U_{\alpha}^{1} \cap U_{\beta}^{1} \neq \emptyset .
$$

This definition of nested open sets is natural because we shall see that in the gluing procedure, we need to shrink the open sets each time we try to glue gauges on overlapping open patches. Let $\left\{U_{\alpha}^{i}\right\}_{\alpha \in I, 1 \leq i \leq K}$ be a $(c, K)$-uniform covering of $M$. We shall define integers $i_{\beta}^{\alpha}$ for all pairs $(\alpha, \beta)$ satisfying $\alpha \geq \beta$. First we define for any $\alpha \in I, i_{\alpha}^{\alpha}=1$. Then we define inductively for $\alpha>\beta$ that

$$
i_{\beta}^{\alpha}= \begin{cases}i_{\beta}^{\alpha-1}+1, & \text { if } U_{\beta}^{i_{\beta}^{\alpha-1}} \cap U_{\alpha}^{1} \neq \emptyset \\ i_{\beta}^{\alpha-1}, & \text { otherwise. }\end{cases}
$$


Note that because of 3) in the definition, for any $\alpha \in I, 1=i_{\alpha}^{\alpha} \leq i_{\alpha}^{\alpha+1} \leq$ $\cdots \leq K$, and the increasing sequence stabilizes to an integer, which we will denote by $i_{\alpha}$.

Definition. We call a $(c, K)$-uniform nested covering $\left\{U_{\alpha}^{i}\right\}_{\alpha \in I, 1 \leq i \leq K}$ of $M$ a $\operatorname{good}(c, K)$-uniform nested covering if there exist functions $\psi_{\alpha} \in C^{\infty}\left(U_{\alpha}^{1}\right)$ such that for each $\alpha \in I$,

$$
\begin{aligned}
& \psi_{\alpha} \equiv 1, \quad \text { on } U_{\alpha}^{1} \cap\left(\bigcup_{\beta<\alpha} U_{\beta}^{i_{\beta}^{\alpha}}\right), \\
& \psi_{\alpha} \equiv 0, \quad \text { on } U_{\alpha}^{1} \backslash\left(\bigcup_{\beta<\alpha} U_{\beta}^{i_{\beta}^{\alpha-1}}\right), \\
& 0 \leq \psi_{\alpha} \leq 1, \quad r_{\alpha}\left|\nabla \psi_{\alpha}\right| \leq c, \quad \text { on } U_{\alpha}^{1}, \text { where } r_{\alpha}=\operatorname{diam}\left(U_{\alpha}^{1}\right) .
\end{aligned}
$$

We recall that a collection of transition functions $\left\{g_{\alpha \beta}\right\}$ with respect to an open covering $\left\{U_{\alpha}\right\}$ of $M$ consist of functions $g_{\alpha \beta}: U_{\alpha} \cap U_{\beta} \rightarrow G$ on $U_{\alpha} \cap U_{\beta} \neq \emptyset$ such that:

1) $g_{\alpha \beta} g_{\beta \alpha}=\mathrm{Id}$, on $U_{\alpha} \cap U_{\beta}$,

2) $g_{\alpha \beta} g_{\beta \gamma} g_{\gamma \alpha}=\mathrm{Id}$, on $U_{\alpha} \cap U_{\beta} \cap U_{\gamma}$.

Definition. We call a collection of transition functions $g_{\alpha \beta} \in C^{1}\left(U_{\alpha} \cap\right.$ $\left.U_{\beta}, G\right)$ a collection of $\delta$-small transition functions with respect to a covering $\left\{U_{\alpha}\right\}_{\alpha \in I}$ with length scales $\left\{r_{\alpha}\right\}_{\alpha \in I}$ if, setting $r_{\alpha}=\operatorname{diam}\left(U_{\alpha}\right)$, we have

$$
\left|g_{\alpha \beta}-\mathrm{Id}\right|+r_{\alpha}\left|\nabla g_{\alpha \beta}\right| \leq \delta r_{\alpha}, \quad \text { on } U_{\alpha} \cap U_{\beta} .
$$

The above technical definitions will enable us to track the bounds effectively in the gluing procedure.

Lemma 1. For any $c_{0}>0$ and $K$ a positive integer, there exists $\delta_{0}=$ $\delta_{0}\left(c_{0}, K, \operatorname{diam}(M), G\right)>0$ such that if $\delta<\delta_{0},\left\{U_{\alpha}^{i}\right\}_{\alpha \in I, 1 \leq i \leq K}$ is a good $\left(c_{0}, K\right)$-uniform nested covering of $M$ and $g_{\alpha \beta}$ is a collection of $\delta$-small transition functions with respect to the covering $\left\{U_{\alpha}^{1}\right\}_{\alpha \in I}$, then there exist a collection of functions $h_{\alpha} \in C^{1}\left(U_{\alpha}^{1}, G\right)$ and a constant $C=C\left(c_{0}, n, K, G\right)>$ 0 such that

$$
\begin{aligned}
& g_{\alpha \beta}=h_{\alpha}^{-1} h_{\beta}, \quad \text { on } U_{\alpha}^{i_{\alpha}} \cap U_{\beta}^{i_{\beta}}, \\
& \left|h_{\alpha}-\mathrm{Id}\right|+r_{\alpha}\left|\nabla h_{\alpha}\right| \leq C \delta r_{\alpha}, \quad \text { on } U_{\alpha}^{1} .
\end{aligned}
$$

Proof. For simplicity, we use $U_{\alpha}$ to denote $U_{\alpha}^{1}$ in the proof. We shall prove by induction on $\alpha \in I$ that there exist $h_{\alpha} \in C^{1}\left(U_{\alpha}, G\right)$ and constants $C(k)=$ $C\left(k, n, c_{0}, G\right)>0$ for $1 \leq k \leq K$ such that for any $\alpha \in I$,

$$
\begin{aligned}
& g_{\beta \gamma}=h_{\beta}^{-1} h_{\gamma}, \quad \text { on } U_{\beta}^{i_{\beta}^{\alpha}} \cap U_{\gamma}^{i_{\gamma}^{\alpha}}, \beta, \gamma \leq \alpha, \\
& \left|h_{\alpha}(x)-\mathrm{Id}\right|+r_{\alpha}\left|\nabla h_{\alpha}(x)\right| \leq C\left(l_{x}^{\alpha}\right) \delta, \quad \forall x \in U_{\alpha},
\end{aligned}
$$


where $l_{x}^{\alpha}=\#\left\{\beta \leq \alpha: x \in U_{\beta}\right\} \leq K$. We note that if (18) and (19) hold for all $\alpha \in I$, then by taking $C=\max _{1 \leq k \leq K} C(k)$, (16) and (17) will follow immediately.

Let $\alpha_{0}$ be the smallest element of $I$, we define $h_{\alpha_{0}}(x)=\operatorname{Id} \in G$ for $x \in U_{\alpha_{0}}$. Then (18) and (19) are trivially satisfied for $\alpha=\alpha_{0}$ (for any value of $C(1)>0)$. Now suppose that we have defined $h_{\beta} \in C^{1}\left(U_{\beta}, G\right)$ for all $\beta<\alpha$ such that (18) and (19) hold for indices $<\alpha$.

We define

(20) $h_{\alpha}(x)=\exp \left(\psi_{\alpha}(x) \exp ^{-1}\left(h_{\beta}(x) g_{\beta \alpha}(x)\right)\right), \quad \forall x \in U_{\alpha} \cap U_{\beta}^{i_{\beta}^{\alpha-1}}, \forall \beta<\alpha$,

$$
h_{\alpha}(x)=\mathrm{Id}, \quad \forall x \in U_{\alpha} \backslash\left(\bigcup_{\beta<\alpha} U_{\beta}^{i_{\beta}^{\alpha-1}}\right) .
$$

We note that by the induction hypothesis, if $\beta<\alpha$, then $\mid h_{\beta}(x) g_{\beta \alpha}(x)-$ Id $\mid \leq C \delta r_{\beta} \leq C \delta$ and $\left|\phi_{\alpha}(x)\right| \leq 1$ by (14). Hence if $\delta$ is sufficiently small, then the expression in (20) involving exp and $\exp ^{-1}$ in (20) is meaningful. The definition in (20) is unambiguous for different choices of $\beta$ because of the induction hypothesis (18) for indices $\beta, \gamma<\alpha$ and the assumption that $\left\{g_{\alpha \beta}\right\}$ are transition functions. We note that the definition (20) and (21) determine a well-defined $h_{\alpha} \in C^{1}\left(U_{\alpha}, G\right)$ because of (12) and (13). It follows easily by (12) and (20), and the fact that $g_{\beta \gamma}$ are transition functions that (18) holds for all $\beta, \gamma \leq \alpha$.

If $x \in U_{\alpha} \cap U_{\beta}^{i_{\beta}^{\alpha-1}}$ with $\beta<\alpha$, then by (14), (15), (20), (21) and the induction hypothesis, there exists a constant $C_{1}=C_{1}\left(n, c_{0}\right)>0$, such that,

$$
\begin{aligned}
\left|h_{\alpha}(x)-\mathrm{Id}\right| & \leq C_{1}\left|h_{\beta}(x) g_{\beta \alpha}(x)-\mathrm{Id}\right| \leq C_{1}\left(1+C\left(l^{\alpha-1}\right)\right) \delta r_{\alpha}, \\
r_{\alpha}\left|\nabla h_{\alpha}(x)\right| & \leq C_{1} r_{\alpha}\left(\left|\nabla \psi_{\alpha}(x)\right|\left|h_{\beta}(x) g_{\beta \alpha}(x)-\mathrm{Id}\right|+\left|\nabla h_{\beta}(x)\right|+\left|\nabla g_{\beta \alpha}(x)\right|\right) \\
& \leq C_{1}\left(1+C\left(l_{x}^{\alpha-1}\right)\right) \delta r_{\alpha} .
\end{aligned}
$$

It follows that if at the beginning we define $C(1)=C_{1}$ and inductively define $C(k+1)=C_{1}(1+C(k))$ (these definitions only depend on $n, K$ and $c_{0}$ ), then (19) will be true for $\alpha$. This finishes the induction step and the proof of the lemma.

Proof of Theorem 1 . We shall choose a collection $\widetilde{\mathcal{F}}$ of disjoint open dyadic cubes in $U \backslash S$ step by step in the following way (the Whitney decomposition): At the first step, we divide $U$ into $2^{n}$ congruent disjoint open cubes with edges of length $1 / 2$. For $k \geq 2$, in the $k$-th step, we consider dyadic cubes with edges of length $1 / 2^{k-1}$ which (or a cube containing it) have not been put in $\widetilde{\mathcal{F}}$ in the previous steps; if such a cube $C$ satisfies the following condition:

$$
\operatorname{dist}(C, S) \geq \operatorname{diam}(C),
$$


then we put $C$ in the collection $\widetilde{\mathcal{F}}$; otherwise, we subdivide $C$ into $2^{n}$ congruent disjoint smaller open cubes with edges of length $1 / 2^{k}$. It is easy to see that we obtain a collection of disjoint open cubes $\widetilde{\mathcal{F}}=\left\{C_{\alpha}: \alpha \in I\right\}$ this way and we have

$$
U \cap \bigcup_{\alpha \in I} \bar{C}_{\alpha}=U \backslash S
$$

We then let

$$
\mathcal{F}=\left\{U_{\alpha}=\left(\frac{9}{8} C_{\alpha}\right) \cap U: \alpha \in I\right\},
$$

where $\frac{9}{8} C_{\alpha}$ is the dilation of $C_{\alpha}$ at the center of $C_{\alpha}$ with a factor $\frac{9}{8}$. The collection $\mathcal{F}=\left\{U_{\alpha}\right\}_{\alpha \in I}$ of open sets now satisfies:

1) $\bigcup_{\alpha \in I} U_{\alpha}=U \backslash S$.

2) If $U_{\alpha} \in \mathcal{F}$, then $\operatorname{dist}\left(U_{\alpha}, S\right) \geq \frac{1}{2} \operatorname{diam}\left(U_{\alpha}\right)$.

3) If $U_{\alpha}, U_{\beta} \in \mathcal{F}$, and $U_{\alpha} \cap U_{\beta} \neq \emptyset$, then $\operatorname{diam} U_{\beta} \leq 3 \operatorname{diam}\left(U_{\alpha}\right)$.

4) There exists a number $K=K(n)$, such that $\#\left\{\alpha: x \in U_{\alpha} \in \mathcal{F}\right\} \leq K$.

Let $\lambda(i)=\exp \left(\frac{K+1-i}{K} \log \frac{9}{8}\right)$ for $1 \leq i \leq K$ and define $U_{\alpha}^{i}=\lambda(i) C_{\alpha} \cap U$ for $\alpha \in I$ and $1 \leq i \leq K$. We note that $U_{\alpha}^{1}=U_{\alpha}$ and $\overline{C_{\alpha}} \subset U_{\alpha}^{K}, \bar{U}_{\alpha}^{i+1} \subset U_{\alpha}^{i}$ for $1 \leq i \leq K-1$. It is then easy to check that there exists a constant $c_{0}=c_{0}(n)$ such that $\left\{U_{\alpha}^{i}\right\}_{\alpha \in I, 1 \leq i \leq K}$ is a good $\left(c_{0}, K\right)$-uniform nested covering.

Under the trivialization of the bundle on $U$, the connection may be identified as a matrix valued 1-form $A$ and a gauge transformation can be viewed simply as a function from $U$ to $G$. We fix a point $x_{0} \in U \backslash S$. Let $x_{\alpha}$ be the center of the cube $C_{\alpha}$. For any point $x \in U_{\alpha}$, we let $\gamma_{\alpha}^{x}$ be the shortest geodesic from $x_{\alpha}$ to $x$ inside $U_{\alpha}$ and define $\mu_{\alpha}(x) \in G$ to be the parallel transport of the bundle from $x_{\alpha}$ to $x$ along $\gamma_{\alpha}^{x}$, using the trivialization of the bundle.

Note that $\mu_{\alpha}\left(x_{\alpha}\right)=$ Id. We regard $\mu_{\alpha}^{-1}$ as gauge transformations on $U_{\alpha}$, and denote $\mu_{\alpha}^{-1}(A)$ by $\widetilde{A}_{\alpha}$. We use the normal spherical coordinates $\left\{r, \theta^{i}\right\}_{i=1, \ldots, n-1}$ centered at $x_{\alpha}$, where $r$ is the distance to $x_{\alpha}$. Assume that

$$
\widetilde{A}_{\alpha}=\widetilde{A}_{\alpha, r} d r+\widetilde{A}_{\alpha, i} d \theta^{i}, \quad \text { on } U_{\alpha}
$$

and

$$
F_{\widetilde{A}_{\alpha}}=F_{\alpha, r i} d r \wedge d \theta^{i}+F_{\alpha, i j} d \theta^{i} \wedge d \theta^{j}, \quad \text { on } U_{\alpha} .
$$

Then by the definition of $\mu_{\alpha}$, we have $\widetilde{A}_{\alpha, r} \equiv 0$ on $U_{\alpha}$. Hence

$$
\partial_{r}\left(\widetilde{A}_{\alpha, i}\right)=F_{\alpha, r i}, \quad i=1, \ldots, n-1 .
$$


By integrating (22) and using that $\widetilde{A}_{\alpha}(0)=0$, we have

$$
\begin{aligned}
\left|\widetilde{A}_{\alpha}\right|(x) & \leq C|x| \cdot \int_{0}^{1}\left|F_{\widetilde{A}_{\alpha}}\right|(t x) d t \\
& =C|x| \int_{0}^{1}\left|F_{A}\right|(t x) d t \leq C|x| \varepsilon_{1} \leq C \varepsilon_{1} r_{\alpha} .
\end{aligned}
$$

Remark. The above gauge coming from the parallel transport along a geodesic leading from a central point was introduced by Uhlenbeck in [11]. It may be called the radially flat gauge at $x_{\alpha}$. Properties (22) and (23) were also given in $[\mathbf{1 1}]$.

Since $\operatorname{dim} S<n-2$, we may perturb the geodesic from $x_{\alpha}$ to $x_{0}$ slightly to be disjoint from $S$ and denote the perturbed curve by $l_{\alpha}$. We define $\sigma_{\alpha}(0) \in G$ to be the parallel transport of the bundle from $x_{0}$ to $x_{\alpha}$ along the curve $l_{\alpha}$ and let

$$
\sigma_{\alpha}(x)=\mu_{\alpha}(x) \sigma_{\alpha}(0), \quad \forall x \in U_{\alpha} .
$$

If $U_{\alpha} \cap U_{\beta} \neq \emptyset$, we denote the difference between the gauge transformations $\sigma_{\alpha}$ and $\sigma_{\beta}$ by

$$
g_{\alpha \beta}=\sigma_{\alpha}^{-1} \sigma_{\beta}=\sigma_{a}^{-1}(0) \mu_{\alpha}^{-1}(x) \mu_{\beta}(x) \sigma_{\beta}(0) .
$$

Now assume that $x \in U_{\alpha} \cap U_{\beta}$. Consider the closed curve

$$
\gamma=l_{\alpha}^{-1}\left(\gamma_{\alpha}^{x}\right)^{-1} \gamma_{\beta}^{x} l_{\beta}
$$

We notice that $g_{\alpha \beta}(x) \in G$ represents the parallel transport of the bundle along the closed curve $\gamma$. Because $\operatorname{dim}(S)<n-2$, by perturbation, there exists a triangle $\Delta$ spanning $\gamma$ with $\operatorname{Area}(\Delta) \leq c r_{\alpha}$ for some constant $c=$ $c(n)$ and $\Delta \cap S=\emptyset$. By a well-known relation between holonomy and curvature, we have

$$
\left|g_{\alpha \beta}(x)-\mathrm{Id}\right| \leq \int_{\Delta}\left|F_{A_{\alpha}}(y)\right| d y \leq c r_{\alpha} \varepsilon_{1} .
$$

Now we use $\sigma_{\alpha}$ as gauge transformations on $U_{\alpha}$ and define

$$
\bar{A}_{\alpha}=\sigma_{\alpha}^{-1}(A)=\sigma_{\alpha}(0)^{-1}\left(\widetilde{A}_{\alpha}\right)=\sigma_{\alpha}(0)^{-1} \circ \widetilde{A}_{\alpha} \circ \sigma_{\alpha}(0) .
$$

Then (23) and the compactness of $G$ imply that

$$
\left|\bar{A}_{\alpha}\right|(x) \leq C \varepsilon_{1} r_{\alpha}, \quad \forall x \in U_{\alpha} .
$$

We have by the definition of $g_{\alpha \beta}$ that

$$
d g_{\alpha \beta}=g_{\alpha \beta} \bar{A}_{\beta}-\bar{A}_{\alpha} g_{\alpha \beta} .
$$

It follows from (26) and (27) that

$$
\left|\nabla g_{\alpha \beta}(x)\right| \leq C \varepsilon_{1} r_{\alpha} .
$$


Given any $\delta>0$, if we take $\varepsilon_{1}=\delta / C$, then (25) and (28) imply that $\left\{g_{\alpha \beta}\right\}$ is a $\delta$-small collection of transition functions with respect to the covering $\left\{U_{\alpha}\right\}$ of $U \backslash S$ with length scales $\left\{r_{\alpha}\right\}$. Therefore Lemma 1 applies and gives the correction term $h_{\alpha}$ on each $U_{\alpha}$. Let $V_{\alpha}=U_{\alpha}^{i_{\alpha}}$. The new gauges $\rho_{\alpha}=h_{\alpha} \sigma_{\alpha}^{-1}$ on $V_{\alpha}$ given by the correction of $h_{\alpha}$ now satisfy $\rho_{\alpha}=\rho_{\beta}$ on $V_{\alpha} \cap V_{\beta}$ (because of (16) and (24)). Hence the $\rho_{\alpha}$ 's define a global gauge $\rho$ on $U \backslash S$. We have

$$
\begin{aligned}
|\rho(A)(x)| & =\left|h_{\alpha}\left(\overline{A_{\alpha}}(x)\right)\right|=\left|h_{\alpha} \bar{A}_{\alpha} h_{\alpha}^{-1}-d h_{\alpha} h_{\alpha}^{-1}\right| \\
& \leq C\left(\left|\bar{A}_{\alpha}\right|+\left|\nabla h_{\alpha}\right|\right) \leq C \varepsilon_{1}, \quad \forall x \in V_{\alpha} .
\end{aligned}
$$

By inspecting our gluing procedure, we know that we can actually require $\rho(A)$ to be smooth away from $S$, which implies that $\rho(A)$ is admissible and a weak solution of the Yang-Mills equation. (29) and the assumption that $\left\|F_{A}\right\| \leq \varepsilon_{1}$ implies that

$$
\|A\|_{L_{1}^{p}} \leq C \varepsilon_{1}, \quad \forall p \leq \infty
$$

because $F_{A}=d A+A \wedge A$. Fix $\frac{n}{2}<p<\infty$, if $\varepsilon_{1}$ is sufficiently small, we may apply the implicit function theorem (see Theorem 2.7 in [11]) to obtain an $L_{2}^{p}$ gauge transformation $g$ on $U$ so that the connection $A^{\prime}=g(\rho(A))$ satisfies

$$
d^{*} A^{\prime}=0, \quad \text { on } U, \quad * A^{\prime}=0, \quad \text { on } \partial U
$$

(as (7) and (8) in Theorem 4's proof). In the new gauge, we have $A^{\prime} \in L_{1}^{p}$. $A^{\prime}$ is also the weak solution of

$$
d_{A^{\prime}}^{*} F_{A^{\prime}}=0, \quad \text { on } U,
$$

by the remark following the proof of Proposition 1 . Therefore we obtain the smoothness of the connection by elliptic regularity and finishes the proof of the theorem.

\section{Global removable singularity theorems.}

Before we carry out the Proof of Theorem 2, we shall first prove the following theorem, which is also of independent interest:

Theorem 7. Assume that $M$ is a compact $n$-dimensional manifold. Let $\mathcal{U}=\left\{U_{\alpha}\right\}_{\alpha \in I}$ be a finite open covering of $M$ and $\left\{g_{\alpha \beta}\right\}, g_{\alpha \beta}: U_{\alpha \beta}=U_{\alpha} \cap$ $U_{\beta} \rightarrow G$, be a set of smooth transition functions with respect to $\mathcal{U}$. Then there exist constants $\varepsilon_{6}=\varepsilon_{6}(M, \mathcal{U})>0$ and $C=C(M, \mathcal{U})>0$, such that if

$$
\sup _{\substack{x, y \in U_{\alpha \beta} \\ \alpha, \beta \in I}}\left|g_{\alpha \beta}(x)-g_{\alpha \beta}(y)\right|=\delta \leq \varepsilon_{6},
$$


then there exist a collection of constant transition functions $\left\{g_{\alpha \beta}^{0}\right\}$, a smaller covering $\mathcal{V}=\left\{V_{\alpha}\right\}$ of $M$, with $V_{\alpha} \subset U_{\alpha}$ and $M \subset \cup \mathcal{V}$, and a set of smooth functions $\rho_{\alpha}: V_{\alpha} \rightarrow G$, such that

$$
\rho_{\alpha} g_{\alpha \beta} \rho_{\beta}^{-1}=g_{\alpha \beta}^{0}, \quad \text { on } V_{\alpha} \cap V_{\beta}
$$

and

$$
\sup _{\substack{x \in V_{\alpha} \\ \alpha \in I}}\left|\rho_{\alpha}(x)-\operatorname{Id}\right| \leq C \delta .
$$

In particular, the bundle defined by $\left\{g_{\alpha \beta}\right\}$ is isomorphic to a flat bundle (defined by $\left\{g_{\alpha \beta}^{0}\right\}$ ).

Proof. We first claim that there exists an increasing continuous function $\mu:[0, \infty) \rightarrow[0, \infty)$ with $\mu(0)=0$, depending only on $M$ and $\mathcal{U}$, such that if $\delta$ is defined by the left-hand side of (31), then there exists a collection of constant transition functions $\left\{g_{\alpha \beta}^{0}\right\}$ with respect to the covering $\mathcal{U}$ such that

$$
\sup _{\substack{x \in U_{\alpha} \cap U_{\beta} \\ \alpha, \beta \in I}}\left|g_{\alpha \beta}-g_{\alpha \beta}^{0}(x)\right| \leq \mu(\delta) .
$$

We let $J=\left\{(\alpha, \beta) \mid \alpha<\beta \in I, U_{\alpha} \cap U_{\beta} \neq \emptyset\right\}$ and $K=\{(\alpha, \beta, \gamma) \mid \alpha<\beta<\gamma \in$ $\left.I, U_{\alpha} \cap U_{\beta} \cap U_{\gamma} \neq \emptyset\right\}$. Denote by $G^{J}$ be the Cartesian product of $|J|$ copies of $G$ indexed by $J$, with a general element $a=\left(a_{\alpha \beta}\right),(\alpha, \beta) \in J$. Define $G^{K}$ similarly. We then define a map $\Phi: G^{J} \rightarrow G^{K}$ by

$$
\Phi\left(\left(a_{\alpha \beta}\right)\right)=\left(a_{\alpha \beta} a_{\beta \gamma} a_{\alpha \gamma}^{-1}\right) \in G^{K} \text {, for any } a=\left(a_{\alpha \beta}\right) \in G^{J} .
$$

$\Phi$ is clearly a continuous map. We note that an element $a=\left(a_{\alpha \beta}\right)$ of $G^{K}$ gives a collection of constant transition functions with respect to $\mathcal{U}$ if and only if $\Phi(a)=(\mathrm{Id}, \ldots, \mathrm{Id}):=1 \in G^{K}$, i.e., if and only if $a \in \Phi^{-1}(1)$. Assume that there doesn't exist such a function $\mu$ as claimed above, then there exists $\varepsilon>0$, a sequence $\delta_{i}$ decreasing to 0 and a sequence of sets of transition functions $\left\{g_{\alpha \beta}^{i}\right\}$ with respect to $\mathcal{U}$, such that

$$
\sup _{\substack{x, y \in U_{\alpha \beta} \\ \alpha, \beta \in I}}\left|g_{\alpha \beta}^{i}(x)-g_{\alpha \beta}^{i}(y)\right|=\delta_{i},
$$

and

$$
\sup _{\substack{x \in U_{\alpha} \cap U_{\beta} \\ \alpha, \beta \in I}}\left|g_{\alpha \beta}^{i}(x)-a_{\alpha \beta}\right| \geq \varepsilon, \forall\left(a_{\alpha \beta}\right) \in \Phi^{-1}(1), \forall i .
$$

We fix points $x_{\alpha \beta} \in U_{\alpha} \cap U_{\beta}$. Because $G^{J}$ is compact, we know that the sequence $\left(g_{\alpha \beta}^{i}\left(x_{\alpha \beta}\right)\right) \in G^{J}$ contains a subsequence converging to an element $\left(g_{\alpha \beta}^{0}\right) \in G^{J}$. Now the fact that $\left\{g_{\alpha \beta}^{i}\right\}$ are sets of transition functions imply 
that $\left\{g_{\alpha \beta}^{0}\right\}$ is also a set of transition functions with respect to $\mathcal{U}$, i.e., $\left(g_{\alpha \beta}^{0}\right) \in$ $\Phi^{-1}(1)$. The fact that $g_{\alpha \beta}^{i}\left(x_{\alpha \beta}\right) \rightarrow g_{\alpha \beta}^{0}$ together with (35) imply that

$$
\sup _{\substack{x \in U_{\alpha} \cap U_{\beta} \\ \alpha, \beta \in I}}\left|g_{\alpha \beta}^{i}(x)-g_{\alpha \beta}^{0}\right| \rightarrow 0, \text { as } i \rightarrow \infty .
$$

This clearly contradicts (36) when $i$. Thus the claim is verified.

Now we recall the following proposition by Uhlenbeck (Prop. 3.2 in [12]):

Proposition 2. Let $\left\{g_{\alpha \beta}\right\}$ and $\left\{h_{\alpha \beta}\right\}$ be two sets of smooth transition functions with respect to a covering $\mathcal{U}=\left\{U_{\alpha}\right\}_{\alpha \in I}$ of a compact manifold $M$. There exist constants $\varepsilon_{7}=\varepsilon_{7}(M, \mathcal{U})>0$ and $C=C(M, \mathcal{U})>0$, such that if

$$
\sup _{\substack{x \in U_{\alpha} \cap U_{\beta} \\ \alpha, \beta \in I}}\left|g_{\alpha \beta}(x)-h_{\alpha \beta}(x)\right| \leq \varepsilon_{7},
$$

then there exists a smaller covering $\mathcal{V}=\left\{V_{\alpha}\right\}$ of $M$, with $V_{\alpha} \subset U_{\alpha}$ and $M \subset \cup \mathcal{V}$, and a set of smooth functions $\rho_{\alpha}: V_{\alpha} \rightarrow G$, such that

$$
\rho_{\alpha} g_{\alpha \beta} \rho_{\beta}^{-1}=h_{\alpha \beta}, \quad \text { on } V_{\alpha} \cap V_{\beta}
$$

and

$$
\sup _{\substack{x \in V_{\alpha} \\ \alpha \in I}}\left|\rho_{\alpha}(x)-\operatorname{Id}\right| \leq C \delta .
$$

In particular, the bundle defined by $\left\{g_{\alpha \beta}\right\}$ is smoothly isomorphic to the bundle defined by $\left\{h_{\alpha \beta}\right\}$.

We apply Proposition 2 to $\left\{g_{\alpha \beta}\right\}$ and $\left\{g_{\alpha \beta}^{0}\right\}$ (found by the claim) and immediately see that the theorem holds.

There are some corollaries of Theorem 7 in the following:

Corollary 1. Assume that $M$ is a compact Riemannian n-dimensional manifold. Let $\mathcal{U}=\left\{U_{\alpha}\right\}_{\alpha \in I}$ be a finite open covering of $M$ such that any two points $x, y$ in a nonempty intersection $U_{\alpha} \cap U_{\beta}$ can be connected by a $C^{1}$ curve within $U_{\alpha} \cap U_{\beta}$ with length $\leq l$, a uniform constant, and let $\left\{g_{\alpha \beta}\right\}$ be a set of smooth transition functions with respect to $\mathcal{U}$. Then there exist constants $\varepsilon_{8}=\varepsilon_{8}(M, l, \mathcal{U})>0$ and $C=C(M, \mathcal{U})>0$, such that if

$$
\sup _{\substack{x \in U_{\alpha \beta} \\ \alpha, \beta \in I}}\left|\nabla g_{\alpha \beta}(x)\right|=\delta \leq \varepsilon_{8},
$$

then we have the same conclusions as in Theorem 7. In particular, the bundle defined by $\left\{g_{\alpha \beta}\right\}$ is smoothly isomorphic to a flat bundle.

Proof. We may easily deduce from (40) and the assumptions that the inequality (31) holds if we take $\varepsilon_{8}=\varepsilon_{6} / l$. Then we can apply Theorem 7 here. 
Corollary 2. Assume that $M$ is a compact $n$-dimensional Riemannian manifold and $E$ is a smooth vector bundle over $M$ with a smooth connection $A$ on it. Then there exists $\varepsilon_{9}=\varepsilon_{9}(M)>0$, such that if

$$
\left\|F_{A}\right\|_{L^{\infty}(M)} \leq \varepsilon_{9},
$$

then $E$ is smoothly isomorphic to a flat bundle.

Proof. We cover $M$ with coordinate balls $\left\{U_{\alpha}\right\}$, such that any two points $x, y$ in a nonempty intersection $U_{\alpha} \cap U_{\beta}$ can be connected by a $C^{1}$ curve within $U_{\alpha} \cap U_{\beta}$ with length $\leq \operatorname{diam}(M)$. Let $\phi_{\alpha}:\left.E\right|_{U_{\alpha}} \rightarrow B_{1}(0) \times \mathbb{R}^{l}$ be trivializations on $U_{\alpha}$ and $A_{\alpha}$ be the $\mathcal{G}$-valued 1-form on $U_{\alpha}$ corresponding to $A$ under $\phi_{\alpha}$. We use the radially flat gauge of $A$ on $U_{\alpha}$, i.e., we find $h_{\alpha}: U_{\alpha} \rightarrow G$ such that $h_{\alpha}\left(A_{\alpha}\right)(0)=0$ and $h_{\alpha}\left(\partial_{r}\right)=0$ under the local coordinates $U_{\alpha} \cong B_{1}(0) \subset \mathbb{R}^{n}$. We have from (23) that

$$
\left|h_{\alpha}\left(A_{\alpha}\right)(x)\right| \leq C|| F_{A} \|_{L^{\infty}(M)} \leq C \varepsilon_{9}, \forall x \in U_{\alpha} .
$$

We define $h_{\alpha \beta}=h_{\alpha} \phi_{\alpha} \phi_{\beta}^{-1} h_{\beta}^{-1}$ on $U_{\alpha} \cap U_{\beta}$ and we can check that $\left\{h_{\alpha \beta}\right\}$ is a set of transition functions. Now we have

$$
d h_{\alpha \beta}=h_{\alpha}\left(A_{\alpha}\right) \circ h_{\alpha \beta}-h_{\alpha \beta} \circ\left(h_{\beta}\left(A_{\alpha}\right)\right)
$$

and hence from (42), we have

$$
\left|\nabla h_{\alpha \beta}\right| \leq C \varepsilon_{9} \text {, on } U_{\alpha} \cap U_{\beta} .
$$

Now by taking $\varepsilon_{9}$ sufficiently small we may apply Corollary 1 to establish the theorem.

Proof of Theorem 2. We choose a covering $\mathcal{U}=\left\{U_{\alpha}\right\}$ of $M$ such that each $U_{\alpha}$ is a coordinate cube and the metrics of $E$ and $M$ on $U_{\alpha}$ can be uniformly compared with the product metric and the Euclidean metric. We also require that any two points $x, y$ in a nonempty intersection $U_{\alpha} \cap U_{\beta}$ can be connected by a $C^{1}$ curve within $U_{\alpha} \cap U_{\beta}$ with length $\leq \operatorname{diam}(M)$. If $\varepsilon_{2}$ is taken small, we can now apply Theorem 1 for the connection $A$ on each $U_{\alpha}$ to obtain local gauge transformations $h_{\alpha}: U_{\alpha} \rightarrow G$ such that $h_{\alpha}(A)$ are smooth on $U_{\alpha}$ and furthermore, from the Proof of Theorem 1, we may require that

$$
\left|h_{\alpha}(A)\right| \leq C\left\|F_{A}\right\|_{L^{\infty}(M)} \leq C \varepsilon_{2},
$$

for some uniform constant $C$. Now we define $h_{\alpha \beta}=h_{\alpha} h_{\beta}^{-1} . h_{\alpha \beta}$ must be smooth because it intertwines smooth connections $h_{\alpha}(A)$ and $h_{\beta}(A)$ on $U_{\alpha} \cap U_{\beta}$. We can then follow the lines of the last part in the proof of Cor. 2 to establish that there exist a refinement $\mathcal{V}=\left\{V_{\alpha}\right\}$ of the covering $\mathcal{U}$, a collection of smooth functions $\rho_{\alpha}: V_{\alpha} \rightarrow G$ and a collection of constant transition functions $g_{\alpha \beta}^{0}$ such that

$$
\rho_{\alpha} h_{\alpha \beta} \rho_{\beta}^{-1}=g_{\alpha \beta}^{0} \text {, on } U_{\alpha} \cap U_{\beta} \text {. }
$$


Because every representation of $\pi_{1}(M) \rightarrow G$ is the trivial one, every flat bundle over $M$ is trivial. Hence there exists constants $\lambda_{\alpha} \in G$ such that $g_{\alpha \beta}^{0}=\lambda_{\alpha}^{-1} \lambda_{\beta}$ for any $\alpha, \beta$. It follows that

$$
\lambda_{\alpha} \rho_{\alpha} h_{\alpha}=\lambda_{\beta} \rho_{\beta} h_{\beta}, \text { on } U_{\alpha} \cap U_{\beta} .
$$

Hence we may define a global gauge transformation $g$ by letting $g=\lambda_{\alpha} \rho_{\alpha} h_{\alpha}$, on $U_{\alpha} . g(A)$ is smooth on $M$ and hence $g$ gives the desired smoothing gauge.

Remark. We remark that in Theorem 2 if the original bundle $E$ is not trivial, then although locally the singularity of the connection $A$ is removable, there may not exist a global gauge transformation which makes $A$ smooth on $M$. The following is a simple example of this lack of global smoothing gauge. In fact, this example has something to do with the lack of global smooth gauge transformations on certain bundles. Let $M=S^{2}=\mathbb{C} P^{1}$ with the covering given by $U_{1}=\{z: z \in \mathbb{C}\}$ and $U_{2}=\{w: w \in \mathbb{C}\}$ with the coordinate change given by $z=1 / w$ on $U_{12}=U_{1} \cap U_{2}$. We give a transition function for a line bundle $g_{12}: U_{12} \rightarrow \mathbb{C}^{*}$ via

$$
g_{12}(z)=\frac{z}{|z|} \in \mathbb{C}^{*} \text {. }
$$

Let $E$ be the smooth line bundle determined by $g_{12}$. It is easy to see that $E$ has the same smooth structure as the hyperplane bundle on $\mathbb{P}^{1}$. We then define a connection $A$ on $E$ by letting its local forms be $A_{1}=-i d \theta$ on $U_{1}$ and $A_{2}=0$ on $U_{2}$, where $\theta$ is the usual angle coordinate on $\mathbb{C}$. We can check that $g_{12}\left(A_{1}\right)=A_{2}$ and hence $A$ is a well-defined connection on $E$ with singularity $p=\{z=0\} \in U_{1}$. This singularity of $A$ can be removed on $U_{1}$ as follows. We define $\rho: U_{1} \rightarrow \mathbb{C}$ by $\rho(z)=\bar{z} /|z|$. Then $\rho\left(A_{1}\right)=0$ gives the smoothing of $A_{1}$ on $U_{1}$. However, it is clear from homotopical considerations that $\rho$ cannot be extended to a global gauge transformation smooth on $M-\{p\}$.

We may similarly construct an example of a nontrivial $S U(2)$ bundle on $S^{4}$ with a flat connection $A$ which is singular at one point but does not have a global smoothing gauge. We note that in these examples the bundles don't allow global smooth gauge transformations.

Acknowledgment. The author would like to thank Rick Schoen and Gang Tian for many helpful discussions on this work and the referee for many useful suggestions on the revision of this paper.

\section{References}

[1] H. Nakajima, Removable singularities for Yang-Mills connections in higher dimensions, J. Fac. Sci. Univ. Tokyo Sect. IA Math., 34(2) (1987), 299-307, MR 89d:58029, Zbl 0637.58026 . 
[2] _ Compactness of the moduli space of Yang-Mills connections in higher dimensions, J. Math. Soc. Japan, 40 (1988), MR 89g:58050, Zbl 0647.53030.

[3] T.H. Otway, Higher-order singularities in coupled Yang-Mills-Higgs fields, Nonlinear Anal., 15(3) (1990), 239-244, MR 91j:58044, Zbl 0714.58049.

[4] T. Parker, Gauge theories on four-dimensional Riemannian manifolds, Comm. Math. Phys., 85(4) (1982), 563-602, MR 84b:58036, Zbl 0502.53022.

[5] J. Råde, Singular Yang-Mills fields, local theory, II, J. Reine Angew. Math., 456 (1994), 197-219, MR 95j:58031, Zbl 0830.53024.

[6] L.M. Sibner, The isolated point singularity problem for the coupled Yang-Mills equations in higher dimensions, Math. Ann., 271(1) (1985), 125-131, MR 86g:58038, Zbl 0558.35073.

[7] _ Removable singularities of Yang-Mills fields in $R^{3}$, Compositio Math., 53(1) (1984), 91-104, MR 86c:58151, Zbl 0552.58037.

[8] L.M. Sibner and R.J. Sibner, Classification of singular Sobolev connections by their holonomy, Comm. Math. Phys., 144(2) (1992), 337-350, MR 93a:58042, Zbl 0747.53024.

[9] T. Tao and G. Tian, A singualarity removal theorem for Yang-Mills fields in higher dimensions. Preprint.

[10] G. Tian, Gauge theory and calibrated geometry, I, Ann. of Math. (2), 151(1) (2000), 193-268, MR 2000m:53074, Zbl 0957.58013.

[11] K.K. Uhlenbeck, Removable singularities in Yang-Mills fields, Comm. Math. Phys., 83(1) (1982), 11-29, MR 83e:53034, Zbl 0491.58032.

[12] Connections with $L^{p}$ bounds on curvature, Comm. Math. Phys., 83(1) (1982), 31-42, MR 83e:53035, Zbl 0499.58019.

Received November 27, 2001 and revised May 19, 2002. Research partially supported by NSF Grant DMS-0104163.

Department of Mathematics

STANFORD UNIVERSITY

STANFORD, CA 94305

E-mail address: byang@math.stanford.edu 